\title{
DOMAIN DECOMPOSITION APPROACH FOR NEAR-WALL TURBULENCE MODELING
}

\author{
Sergey V. Utyuzhnikov ${ }^{1},{ }^{2}$ \\ ${ }^{1}$ School of MACE, University of Manchester, \\ Manchester, M13 9PL, UK \\ e-mail: s.utyuzhnikov@manchester.ac.uk \\ ${ }^{2}$ Moscow Institute of Physics and Technology, \\ Dolgoprudny 141700, Russia
}

Keywords: Near-wall Turbulence Modeling, Domain Decomposition, Wall Functions, Reynolds Models.

\begin{abstract}
.
Turbulence modeling leads to a multiscale problem in which there is a very thin boundary layer that requires most computational time for its resolution. The paper addresses a novel approach based on non-overlapping domain decomposition. It allows us to avoid calculations of the region with high gradients in the vicinity of the wall while retaining sufficient overall accuracy. The technique is introduced in application to low-Reynolds number RANS models. The domain decomposition is achieved via the transfer of the boundary condition from the wall to an interface boundary. If the governing equations in the inner domain are simplified, then the interface boundary conditions are of Robin type. These boundary conditions can be obtained in an analytical form despite the fact that they are nonlinear. Possible ways to achieve a reasonable trade-off between efficiency and accuracy are discussed.

The obtained interface boundary conditions are mesh-independent. They can be used to avoid computationally expensive resolution of a high-gradient region near the wall. Moreover, once the solution is constructed in the outer region, the near-wall profile can be restored if required. In two extreme cases, if the interface boundary is too close to the wall or too far from $i t$, the so-constructed solution to the problem automatically corresponds to the low-and highReynolds number RANS models, respectively. Different applications are considered including unsteady problems and complex geometries. It is shown that in comparison to the low-Reynolds number models the near-wall domain decomposition approach allows the computational time to be reduced by one order of magnitude whilst practically retaining the accuracy.

The developed approach proved to be quite robust and relatively universal. It does not contain any tuning parameters. The technique might be extended to other multiscale problems.
\end{abstract}




\section{INTRODUCTION}

Numerical modeling turbulent flows near walls is still computationally very expensive. The problem is that nearby the wall there is a very thin laminar boundary layer due to the no-slip boundary condition and damping effect of the wall. The thickness of the laminar sublayer is about $1 \%$ of the entire thickness of the boundary layer. However, resolution of this region takes up to $90 \%$ of the total computational time [17] because of high gradients of the solution. One possible way to avoid this problem is the use of high Reynolds number (HRN) models. In this case the flow structure above the laminar sublayer is deliberately not resolved. Instead of this the solution is represented by approximate boundary conditions which are usually set at the nearest to the wall cell. Such Dirichlet boundary conditions are often called the wall functions. In the core flow the governing Reynolds Averaged Navier-Stokes rquations (RANS) do not depend on the distance to the wall in contrast to the original low Reynolds number (LRN) RANS models. Thus, all information on the wall is supposed to be included in the wall functions. As noted in [11], this approach should be treated as a domain decomposition in which the solution in the inner near-wall layer is replaced by the wall functions.

As mentioned above, the wall functions represent an approximate solution in the inner layer. They can be obtained in one way or another one. First wall functions were based on the analytical solution for a plate [18], [12] and its semi-empirical modifications (see e.g. [13], [14]) to make them more universal. More advanced wall functions such as [15], [16] are not restricted by approximate local solutions. They take into account the solution in the outer (core) region via iterations. The solution in the inner region is obtained by solving boundary layer equations either numerically or analytically. In the latter case the solution of the governing equations is supposed to be approximate. In this case a domain decomposition can be realized with a Dirichlet-Dirichlet (D-D) iterative algorithm [19].

As shown in [9], the interface boundary condition (IBC) between the inner and outer regions can be obtained via transfer the boundary condition from the wall to the interface boundary. In this approach, the wall boundary conditions and governing equations are equivalently replaced by the IBC [10]. If the governing equations in the inner layer are of boundary-layer type and locally one-dimensional, the transfer of boundary condition can be exactly realized [10]. One can prove that in the case of a Dirichlet boundary condition set at the wall the IBC is always of Robin type. In a more general formulation the IBC must be nonlocal in both space and time [7], [3]. In [7] for a model equation it is demonstrated that if the flow along the wall changes rapidly enough, then the IBC must be nonlocal, otherwise essential effects can be lost. In turn, in [3] for a model unsteady equation it is shown that if the solution is essentially unsteady, then the IBC must be nonlocal in time and contain a memory term. In [4] the approach was successfully applied for modeling laminar-turbulent transition. In general the opportunity to replace the solution in the inner region by an interface pseudo-differential equation follows from the theory of generalized Calderón-Ryaben'kii's potentials that are proved to be projections [8]. The technique of boundary condition transfer can be applied to the LRN models straightforward [5]. In this case the distance between the interface boundary and wall is the parameter reflecting the trade-off between the accuracy and time consumption [1]. As shown in [1], in comparison with the LRN models the near-wall domain decomposition approach can reduce the computational time by one order of magnitude whilst the accuracy dropping by about $1 \%$. In [1] the near-wall domain decomposition technique was implemented with different RANS models and successfully applied to a few industrial problems.

In the current paper the approach is described in a general formulation. The algorithm of 
realization is given in detail. The approach is compared against standard methods. Examples of practical applications are provided. Some prospectives for future realization are also discussed.

\section{NEAR-WALL DOMAIN DECOMPOSITION}

Consider first a domain decomposition for a 1D non-linear equation defined in an interval $\Omega:=\left[0, y_{e}\right]:$

$$
\begin{gathered}
\mathcal{L}_{y}(U)=f, \\
U(0)=U_{0}, \\
U\left(y_{e}\right)=U_{1} .
\end{gathered}
$$

where $\mathcal{L}_{y}$ is a non-linear differential operator of second order.

Next, we split the interval $\Omega$ into two intervals $\Omega^{-}:=\left[0, y^{*}\right]$ and $\Omega^{+}:=\left[y^{*}, y_{e}\right]$. To transfer the boundary condition from the wall $(y=0)$ to $y=y^{*}$ one can use the nonlinear potentials introduced in [6]. The IBC is formulated as follows:

$$
U_{y}=\Psi(U, f) .
$$

Although being exact, such a boundary condition is essentially nonlinear and not easy to be used.

To simplify the IBC, presume that in the inner region the governing equation is of boundarylayer type: $\mathcal{L}_{y}(U) \equiv \frac{d}{d y}(\mu U)$, where $\mu$ is the efficient viscosity coefficient, then the IBC is reduced to a Robin boundary condition formulated at $y^{*}$ [10]:

$$
U^{\prime}=\frac{U-U_{0}}{I_{1}}+\frac{I_{1} I_{S 1}-I_{S 2}}{\mu^{*} I_{1}},
$$

where $I_{1}=\int_{0}^{y^{*}} \frac{\mu^{*}}{\mu} d y, I_{S 1}=\int_{0}^{y^{*}} f d y, I_{S 2}=\int_{0}^{y^{*}} \frac{\mu^{*}}{\mu} \int_{0}^{y^{\prime}} f d y d y^{\prime}, \mu^{*}=\mu\left(y^{*}\right)$.

In 1D case the boundary equation (4) fully replaces the solution of the problem in the inner region $\Omega^{-}$for the outer region $\Omega^{+}$. This means the solution of boundary value problem $(1)$, (2) must satisfy equation (4). In turn, it is easy to prove that condition (4) is unique. In the linear case equation (4) would be totally independent of the solution in the outer region. In our consideration the dependance is weak. It is only revealed via the turbulent viscosity coefficient that depends on the solution in the entire region. It is worth noting that it is impossible to obtain the solution in the outer region without the solution in the inner region. However, it is possible to transfer the boundary condition from the wall to the interface boundary without knowledge of the solution in the outer region even in the nonlinear formulation. In turn, it is impossible to obtain the solution in $\Omega^{-}$with boundary condition 4 because this condition is the consequence of the governing equation and wall boundary condition.

The IBC are formulated in a universal form (4) for all variables but the normal to the wall velocity. The problem is that the conservation law of mass is not directly used in the IBC. It might be violated if the approximation does not guarantee the integral conservation law in the inner region as a whole. In this case a Dirichlet boundary condition for the normal velocity suggested in [1] can be used.

To obtain the solution in the entire region via the near-wall domain decomposition, the procedure is the following. First, one should obtain the solution in the outer region $\Omega^{+}$with boundary 
condition (4) at the left-hand side. Next, a boundary value problem (BVP) is to be solved in the inner region $\Omega^{+}$with the Dirichlet boundary condition at the right-hand side. This boundary condition becomes known once BVP in the outer region has been solved. It is not always necessary to solve the BVP in the inner region since the friction at the wall is immediately related with the friction $\tau_{w}$ at the interface boundary:

$$
\tau_{w}=\mu\left(y^{*}\right) \frac{d U}{d y}-\int_{0}^{y^{*}} f d y
$$

In boundary equation (4), $\mu$ represents the sum of laminar and turbulent viscosity coefficients. It is to be noted that the IBC (4) is exact even in the nonlinear case when the turbulent viscosity coefficient, $\mu_{t}$, depends on the solution. To simplify the use of boundary condition (4), one can use available approximations for $\mu_{t}$ nearby the wall. Some examples are given in [1]. Apart from a piece-wise linear approximation used in [15] for the analytical wall functions, the others are nonlinear because they depend on the wall friction.

Thus, the entire algorithm is as follows:

$1^{0}$. Select an approximation for the near-wall turbulent viscosity coefficient $\mu_{t}$.

$2^{0}$. Select an initial approximation for $\tau_{w}$ (for nonlinear approximations of $\mu_{t}$ ).

$3^{0}$. Calculate IBC (4).

$4^{0}$. Solve BVP in the outer region with the IBC.

$5^{0}$. Update $\tau_{w}$ via (5)

$6^{0}$. Update the near-wall turbulent viscosity profile.

$7^{0}$. Repeat the procedure from stage 3 .

If necessary, in stage $5 \mathrm{BVP}$ in the inner region can be solved with Dirichlet boundary conditions at $y^{*}$ obtained from the solution in the outer region. The governing equations in the inner region are sufficient to calculate the turbulent viscosity coefficient. In this case, stage 1 can be avoided. In multidimensional case, the IBC can be nonlocal [7]. Alternatively, in the inner region the governing equations can be of boundary-layer type because nearby the wall the normal derivatives are predominant. In this case, the transfer of boundary conditions can be locally one-dimensional.

It is interesting to compare the approach against the advanced-wall-function techniques [15], [16]. First, one should note that the wall functions are only applied to the HRN models. The key point is that the interaction between the inner and outer regions is carried out via D-D iterative methods. As noted above, the IBC (4) is unique regardless the approach. This means in the D-D algorithms with wall functions the derivative $U^{\prime}$ is taken from the previous iteration. Since it is a leading term, the number of iterations should significantly increase. In turn, this damages the robustness. In the linear case the near-wall domain decomposition would be exact and not require iterations in contrast to the $\mathrm{D}$-D algorithm. In the linear case the near-wall domain decomposition would be exact and not require any iterations in contrast to the D-D algorithm. It is to be noted that the IBC are mesh independent. They are formulated in a differential form. The coefficients in the IBC can be calculated either analytically or numerically. In the latter case the mesh in the inner region is not related with the mesh in the outer region at all. Therefore, the IBC can be easily implemented in a code via a separate routine.

As mentioned above, the introduced technique can be applied to LRN RANS models straightforward. If in the inner region the problem is solved approximately, then there is a trade-off between the computational time and accuracy. Consider two extreme cases. If $y^{*}$ tends to zero, then the solution model becomes equivalent to the LRN model. However, the effect of the approach vanishes. In turn, if $y^{*}$ is big enough, then the computational time should significantly 
drop by the cost of accuracy. In this case the approach becomes similar to HRN models with respect to the accuracy of prediction because the laminar sublayer region is not resolved.

The approach represents an approximate non-overlapping near-wall domain decomposition (NDD). It is natural to expect that the computational time with the NDD should significantly be less than that without it. The justification is the following. The computational time to inverse an operator is proportional to its condition number. One can show that in the case of the NavierStokes equations the condition number is approximately proportional to the ratio between the maximal and minimal space steps squared. For the sake of simplicity, consider a mesh which is adaptive in the direction normal to the wall. Obviously, the condition number in the outer region should decrease rapidly as $y^{*}$ increases so should do the computation time. In turn, since the inner region is only approximately resolved, the error of prediction should inevitably decrease.

The trade-off between the computational time and accuracy was investigated in [1] for a two-dimensional asymmetric diffuser test case. The sketch of the diffuser is given in Fig. 1.

In Figure 2 a relative computational time is shown for the SST model [20]. Here, $e$ represents a relative error in computation of the skin friction. One can see that the near-wall domain decomposition can reduce the computation time by one order of magnitude whilst the error is just about one percent. The approach with the wall functions requires approximately the same time but the error is above $100 \%$ in this case. The results are shown for the SST model (LRN), NDD and scalable wall functions [13] (HRN).

The skin friction distribution along the inclined wall at different $y^{*}$ is given in Figure 3. The solid dot line represents the experimental results by Buice and Eaton [21]. Computational results demonstrate that dependence of the solution on $y^{*}$ is not essentially sensitive to $y^{*}$ [10], [5], [1], [2] if $y^{*}$ is small enough. The variation of $y^{*}$ by one order of magnitude leads to the change of the solution as much as a few percent. Thus, a reasonable value of $y^{*}$ can be easily evaluated from either general information of the solution to be obtained or preliminary calculations on a coarse mesh.

In the future the approach can be extended to unsteady problems and nonlocal effects. Preliminary development of this technique was carried out in [3] and [5], respectively.

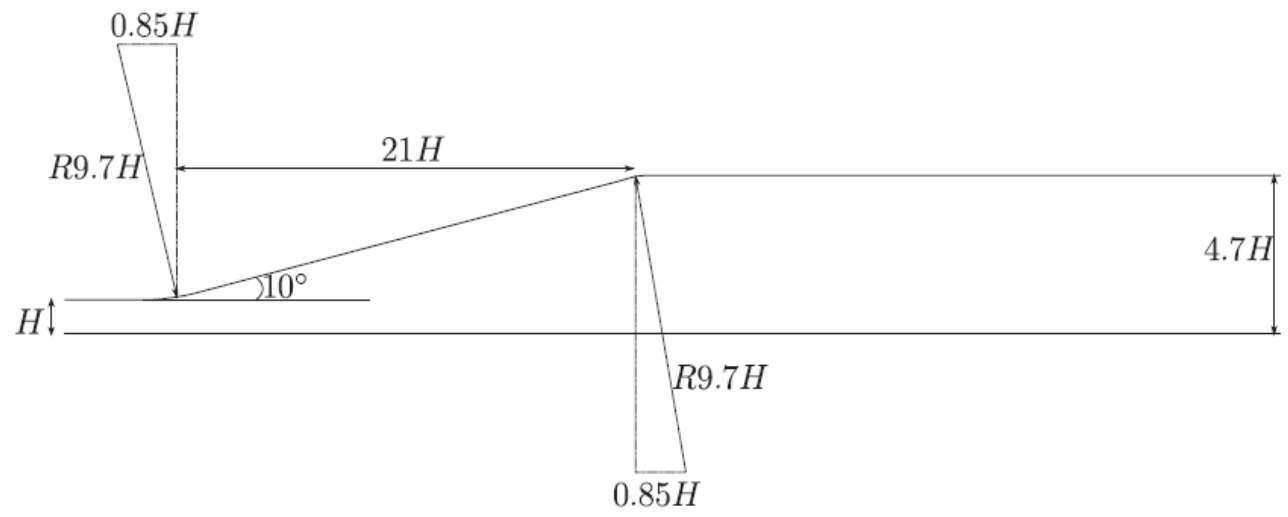

Figure 1: Two-dimensional asymmetric diffuser. 


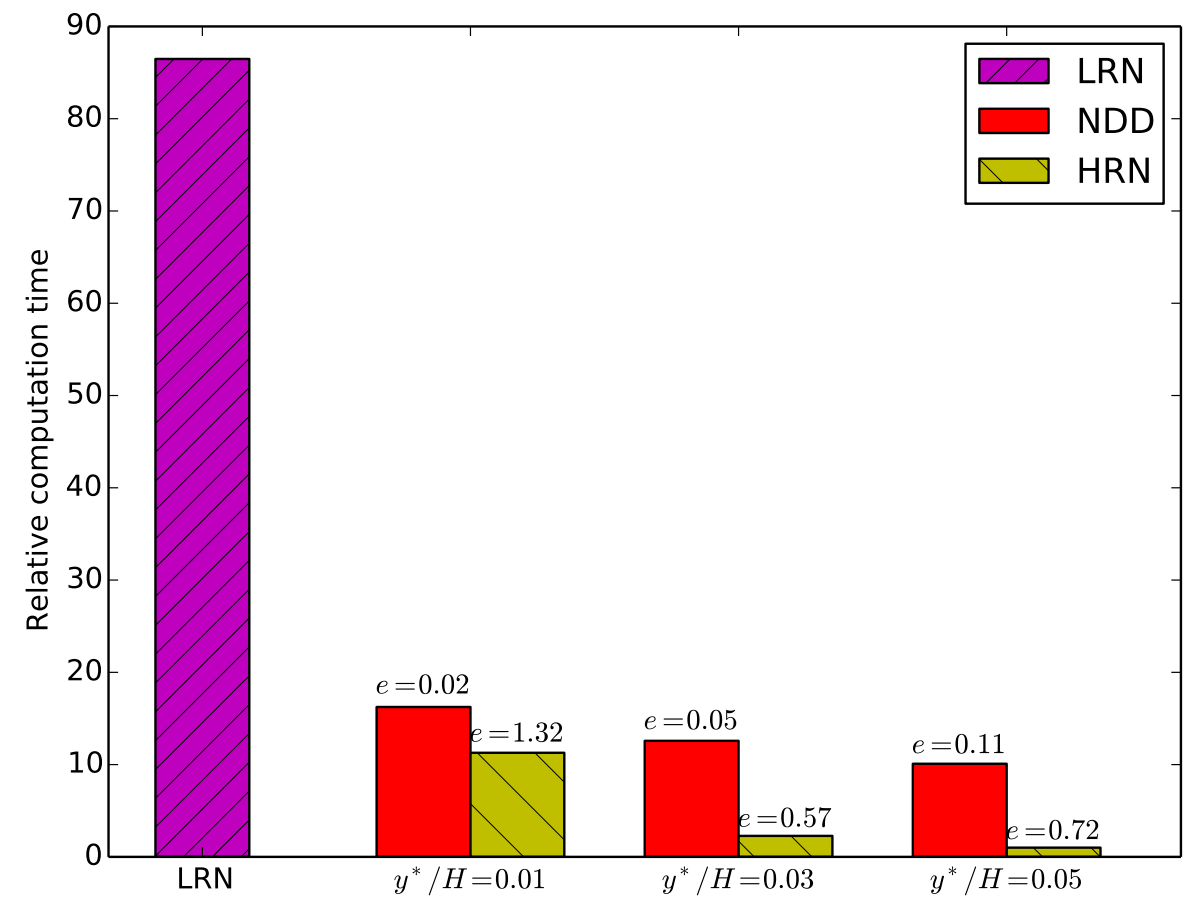

Figure 2: Trade-off between the accuracy and computational time for a two-dimensional asymmetric diffuser [1]. Low-Reynolds number SST model (LRN) [20], near-wall domain decomposition (HRN) and scalable wall functions (HRN) are used. $e$ is the relative error of prediction with respect to LRN.

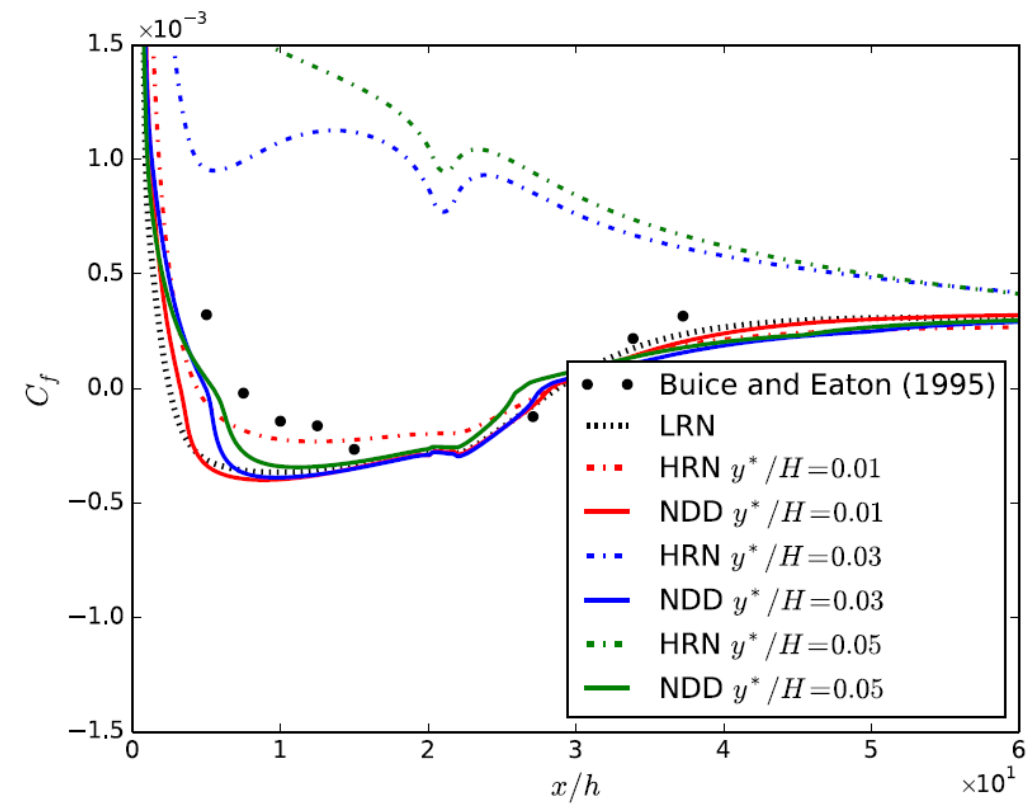

Figure 3: The skin friction distribution along the inclined wall of the diffuser for different $y^{*}$ [1]. 


\section{References}

[1] A. Jones, S.V. Utyuzhnikov, Application of a near-wall domain decomposition method to turbulent flows with heat transfer. Computers \& Fluids, 119, 87-100, 2015.

[2] A. Jones, S.V. Utyuzhnikov, A near-wall domain decomposition approach in application to turbulent flow in a diffuser. Applied Mathematical Modelling, 40, (1): 329-342. 2016.

[3] S.V. Utyuzhnikov, Towards development of unsteady near-wall interface boundary conditions for turbulence modelling. Comp. Phys. Communic., 185, 11, 2879-2884, 2014.

[4] Aleksin, V.A. Aleksin, S.V. Utyuzhnikov, Implementation of near-wall boundary conditions for modelling boundary layers with free-stream turbulence. Applied Mathematical Modeling, 38, 14, 3591-3606, 2014.

[5] S.V. Utyuzhnikov, Interface boundary conditions in near-wall turbulence modeling. Computers \& Fluids, 68, 186-191, 2012.

[6] S.V. Utyuzhnikov, Nonlinear Problem of Active Sound Control. J. of Computational and Applied Mathematics, textbf234 (1): 215-223, 2010.

[7] S.V. Utyuzhnikov, Domain decomposition for near-wall turbulent flows. Computers \& Fluids, 38, (9), 1710-1717, 2009.

[8] S.V. Utyuzhnikov, Generalized Calderon-Ryabenkiis potentials. IMA J. of Applied Mathematics, 74, (1): 128-148, 2009.

[9] S.V. Utyuzhnikov, The method of boundary condition transfer in application to modeling near-wall turbulent flows. Computers \& Fluids, 35, (10), 1193-1204, 2006.

[10] S.V. Utyuzhnikov, Robin-type wall functions and their numerical implementation. J. Applied and Numerical Mathematics, 58, (10), 1521-1533, 2008.

[11] P.A. Durbin, Limiters and wall treatments in applied turbulence modeling. Fluid Dynamics Research, 41, 1-8, 2009.

[12] B.E. Launder, B.I. Spalding, The numerical computation of turbulent flows. Computer Methods in Applied Mechanics and Engineering, 3, 269-289, 1974.

[13] H. Grotjans, F.R. Menter, Wall functions for industrial applications. K.D. Papailiou eds. Proceedings of Computational Fluid Dynamics'98, ECCOMAS, 1(2), Chichester, UK: John Wiley \& Sons, 1112-1117, 1998.

[14] G. Kalitzin, G. Medic, G. Iaccarino, P.A. Durbin, Near-wall behavior of RANS turbulence models and implications for wall functions. J. Computational Physics, 204, 1, 269-289, 2005.

[15] T.J. Craft, A.V. Gerasimov, H. Iacovides, B.E. Launder, Progress in the generalization of wall-functions treatments. Heat and Fluid Flow, 23, 148-160, 2002.

[16] T.J. Craft, S.E. Gant, H. Iacovides, B.E. Launder, A new wall function strategy for complex turbulent flows. Numerical Heat Transfer, 45, 301-318, 2004. 
[17] T. Kopp, On grid-independence of RANS predictions for aerodynamic flows using modelconsistent universal wall-functions. Proceedings of ECCOMAS CFD'2006, The Netherlands, 2006.

[18] S.V. Patankar, D.B. Spalding, Heat and mass transfer in boundary layers. MorganGrampian Press, London, 1967.

[19] A. Quarteroni and A. Valli, Domain Decomposition Methods for Partial Differential Equations. Clarendon Press, Oxford, 1999.

[20] F.R. Menter, M. Kuntz, R. Langry, Ten years of industrial experience with the SST turbulence model. Turbulence, Heat and Transfer, 4, 625-632, 2003.

[21] C.U. Buice, J.K. Eaton, Experimental investigation of flow through an asymmetric plane diffuser. Centre of Turbulence Research, Annual Research Brief 1995, 117-120, 1995. 